\title{
Smooth Context based Color Transfer
}

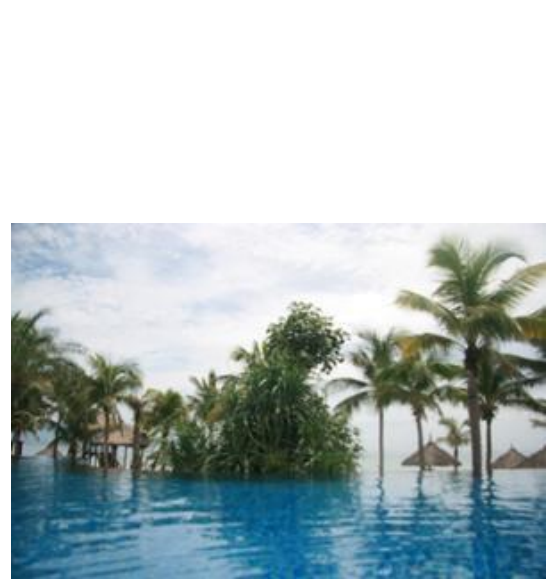

a. Input image

\author{
Dao Nam Anh \\ Department of Information Technology \\ Electric Power University \\ 235 Hoang Quoc Viet road \\ Hanoi, Vietnam
}

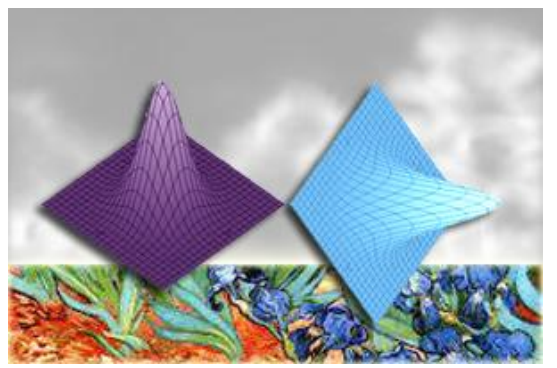

b. Smooth context by bilateral filter on top, and target color at the bottom

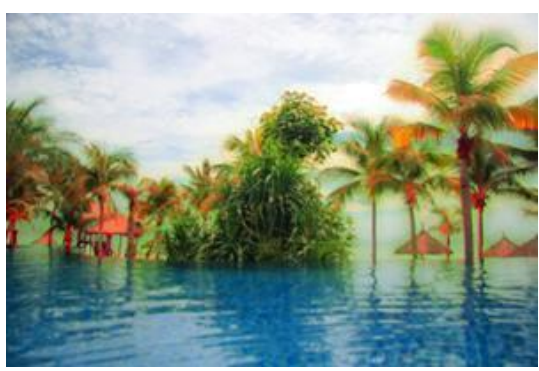

c. Result, $\mathrm{SSIM}=0.9998, \mathrm{t}=4.05$

Figure 1:Color transfer in smooth context

\begin{abstract}
Color transfer is an emerging framework for dealing with ubiquitous color manipulation in media such as documents and images. Despite the notable progress made in the field, there remains a need for designers that can represent the same information in personalization and corresponding to media context. This work presents adaptive color transfer method using cross-disciplinary interaction of semantic context and bilateral filters. Colors in the method are transferred softly in matching with saliency distributed context. Preliminary results show that the framework is highly keeping consistency and promising. Consequently in this work, a solution of tone mapping by color transfer is introduced. Experimental results are further showed pertaining for automatic handling colors and contrast.
\end{abstract}

\section{General Terms}

Pattern Recognition, Algorithms

\section{Keywords}

Context, smooth, color transfer, bilateral filter, saliency, tone mapping

\section{INTRODUCTION}

As digital camera technology has advanced in the past decade, colors manipulation, and color transfer in particular is still fundamental and strong basics for several image analysis applications. The term color transfer is used here specifically to the transferring the color style from the target image to the source image.

Reinhard et al in [1] provides a comprehensive overview of simple solution to impose one image's color characteristics on another, where color correction is achieved by choosing an appropriate source image and applying its characteristic to another image. The use of statistical analysis can greatly simplify the computational load of learning colors, provided it can be successfully matched and optimized for the problem at hand. Recent works by [2], [3], [4], [5] have made great strides in scaling both automatic and custom-handled color transfer, though context consistency of these models remains unremarkable.

In addition, recent saliency findings suggest that salient regions should contain not only the prominent objects but also the parts of the background that convey the context [6]. Such salient regions facilitate effective matching and interpretation of visual information, particularly in the context of capturing spatio-color dependencies.

In fact, if a stimulus is insufficiently salient, sky and water in a scene as in Fig.1a are not changed in color transfer result as in Fig.1c. In this case, saliency map displayed in upper part ofFig.1b is fairly reasonable. Target color as in bottom part asofFig. $1 \mathrm{~b}$ is applied mainly to foreground by the saliency map. The map is smooth but it keeps major features by bilateral filter [9]. The result looks more natural with new color transferred into foreground while keeping background mostly the same. Structural similarity (SSIM) index [7] is 0.9998 for the case.

This work has several technical contributions. Firstly, new algorithm of Smooth Context Based Color Transfer (SCCT) is proposed and analyzed. This is a development of statisticsbased method [8] for color distribution transfer. The method used different color distribution to find mapping relations for color transfer. This method now is combined with contextaware saliency [6] and bilateral filter [9] in our work to produce a new way of color transfer. Secondly, a solution for tone mapping is suggested. This is the practical implementation the SCCT algorithm for high dynamic range images. Though the solution is not fully automatic but it's simple, effective and can produce creative results in artistic styles following artist's reference.

\section{OUTLINE OF PAPER}

In the following sections core concepts are reviewed pertaining to the smooth context based color transfer. The main color transfer mechanisms are discussed along with key metrics and parameters for formulating those mechanisms. Then the experiments are discussed for the McGill Calibrated Color Image Database [10] and results of using SCCT as a solution for the color transfer are demonstrated. High dynamic range images [11] then are tested with tone mapping solution by SCCT. Finally, the article is concluded with discussion and summary of projected future directions for our work.

\section{PRIOR WORK}

On the part to follow the article goals and approach it is helpful to review shortly some major color transfer methods. The task of color transfer still get a lot of attention since handling colors is essential in image analysis. Therefore, in 
the last decades, a lot of work has been focused on the problem of color transfer.

Reinhard et al. [1] raised interest on color transfer by introducing the use of statistical analysis for transfer color between images. The mean and variance of colors in axes $l \alpha \beta$ in the CIELAB color space are imposed in matching metrics. The operation is simple and effective, though it produces slight grain effect. Histogram [12] matching is also simple and fast method. As it does not check continuity of colors between neighborhoods pixels, results may have color distortion.

Another approach is to reduce the high-dimensional Probability Density Function (PDF) matching problem to the one-dimensional PDF matching by Radon Transform[13]in solving N-dimensional PDF for transfer [2], [8]. This method is a non-parametric and it requires low computation costs for matching arbitrary distributions. Color correlation is reduced. This work develops the approach in next section.

Work in [4] proposed gradient-preserving model that consider both color distribution and the scene details. An extended Principal Component Analysis (PCA) based transfer is used to manage the color range, and a minimization scheme is the base to generate color features. Optimal solution of the method requires large computational cost though it preserves the grid structure of gradient meshes.

Remote sensing image fusion using non-separable wavelet frame transform is a solution in [14] for the case, where it fuses high spatial resolution panchromatic image and low spatial resolution multispectral image of the same scene.

Recently, transfer color in 3D space [15] is proposed with scattered point interpolation scheme using moving least squares and strengthen it with a probabilistic modeling. The model can deal with mis-alignments and noise.

Color transfer can be performed by a dominant color idea [3]. The method produces good results when there is consistence between the amount of dominant colors of the target and that of the reference. color distortion may have place when the amount of dominant colors are not balanced.

Visual saliency is a profound challenge for detection of the salient regions of an image. The saliency factor estimated for regions is fairly benefit for object co-segmentation [16], classification [17], retrieval [18] and object tracking [19]. A solution of color transfer by the mean and variance of colors [1] is enforced with saliency map in [20].

Bilateral filter proposed by Tomasi and Manduchi [9] is noniterative, stable and simple method. It takes into account both space and intensity difference from the central pixel to cut off the noise and avoid edges diffusing. The filter is applied to saliency map in our work.

Color transferis crucial for multimedia applications, starting from color correction. Selective color from reference image can be transferred to region where color is distorted [21]. Image in painting is a special case applicable by color transfer where all information including structure data in some regions is lost.

Stylizing images by manipulating color and contrast is the application addressed in [5]. Photos styles are managed by their associated key words and their colors can be changed by styles for consistency with context key words.

Learning abstract categories on color palettes can be used in the application of color transfer, personalization and image reranking [14]. The work suggests to consider combinations of
International Journal of Computer Applications (0975 - 8887)

Volume 116 - No. 15, April 2015

colors or color palettes instead of a single color to learn categorization models.

Contrast transfer is another application of color transfer where pure and high contrast can be replaced by selected color contrast predefined in reference image. In some cases tone mapping can be seen like contrast transfer and it is solved by color transfer. Section 5 of this work presents application of our color transfer algorithm for tone mapping.

\section{CONTEXT-BASED FILTER}

Denote foreground by $\Omega_{f}$ and background by $\Omega_{b}$ for color image $u(x)$ :

$$
\begin{aligned}
& u(x): \Omega \rightarrow \mathfrak{R}^{3} \\
& \Omega=\Omega_{f} \cup \Omega_{b}, \Omega_{f} \cup \Omega_{b}=\varnothing
\end{aligned}
$$

Working in the CIE Lab color space[26] is suggested for saliency detection as the Lab is designed to approximate human vision.

Convert RGB to Lab, and back functions:

$$
\begin{aligned}
& u_{R G B}(x) \rightarrow u_{\text {Lab }}(x) \\
& u_{L a b}(x) \rightarrow u_{R G B}(x)
\end{aligned}
$$

\subsection{Saliency Map}

Saliency detection creates saliency $\operatorname{map} s(x)$ and define $\Omega_{f}$, $\Omega_{b}:$

$$
\begin{aligned}
& s(x): \Omega_{f} \rightarrow \mathfrak{R}^{1} \\
& \Omega_{f}=\{x, s(x)>\tau\}, \Omega_{b}=\Omega / \Omega_{f}
\end{aligned}
$$

According to context aware saliency[6], patch $p(x)$ is defined by pixel $x$ and its surrounding patch, which gives an immediate local context. Let's recall distance between two patches:

$d(p(x), p(y))=\frac{d_{\text {color }}(p(x), p(y))}{1+c(x) * d_{\text {position }}(p(x), p(y))}$

where $d_{c o l o r}$ and $d_{\text {position }}$ are Euclidean distance between the color/positions of patches $p(x)$ and $p(y)$.

Context evaluation $c(x)$ depends on $s(x)$ :

$c(x)=\lambda . s(x)$

\subsection{Bilateral Filter}

Bilateral filter [9] that ignores a part of details and keeps major edges is applied to context $c(x)$ to prevent color distortion and grain effect:

$$
b(\mathrm{x})=\frac{1}{W_{\mathrm{x}}} \sum_{\mathrm{y} \in S} G_{\sigma_{s}}(\|\mathrm{x}-\mathrm{y}\|) G_{\sigma_{r}}(\|c(\mathrm{x})-c(\mathrm{y})\|) c(\mathrm{y})
$$

Filter scales of the spatial $\sigma_{s}$ and intensity $\sigma_{r}$ manage the smooth effect of the filter.

\subsection{Color Transfer}

Input image $u(x)$ and reference image $r(x)$ have their sets of color samples. The distribution transfer problem [2] is to find a differentiable mapping $t$ that transforms the original color PDF $f(u(x))$ into a new color PDF $g(y(x))$ that matches the reference PDF $g(r(x))$ : 


$$
\int^{u} f(u) d u=\int^{t(u)} g(y) d v
$$

The Kullback-Leibler (KL) divergence [22] is used as a measure to quantify the level of matching transformed distribution $f^{(k)}$ :

$D_{K L}(f \| g)=\int_{u} f(u) \ln \left(\frac{f(u)}{g(u)}\right) d u$
International Journal of Computer Applications (0975 - 8887)

Volume 116 - No. 15, April 2015

Having full color transferred $y(x)$ by (10), (11) [2], contextbased color transfer for foreground $\Omega_{f}$ is done by the following regulation:

$v(x)=b(x) \cdot y(x)+(1-b(x)) \cdot u(x)$

To transfer color with context $b(x)$ for background $\Omega_{b}$ :

$v(x)=(1-b(x)) \cdot y(x)+b(x) \cdot u(x)$

Now algorithm can be formulated as follows.

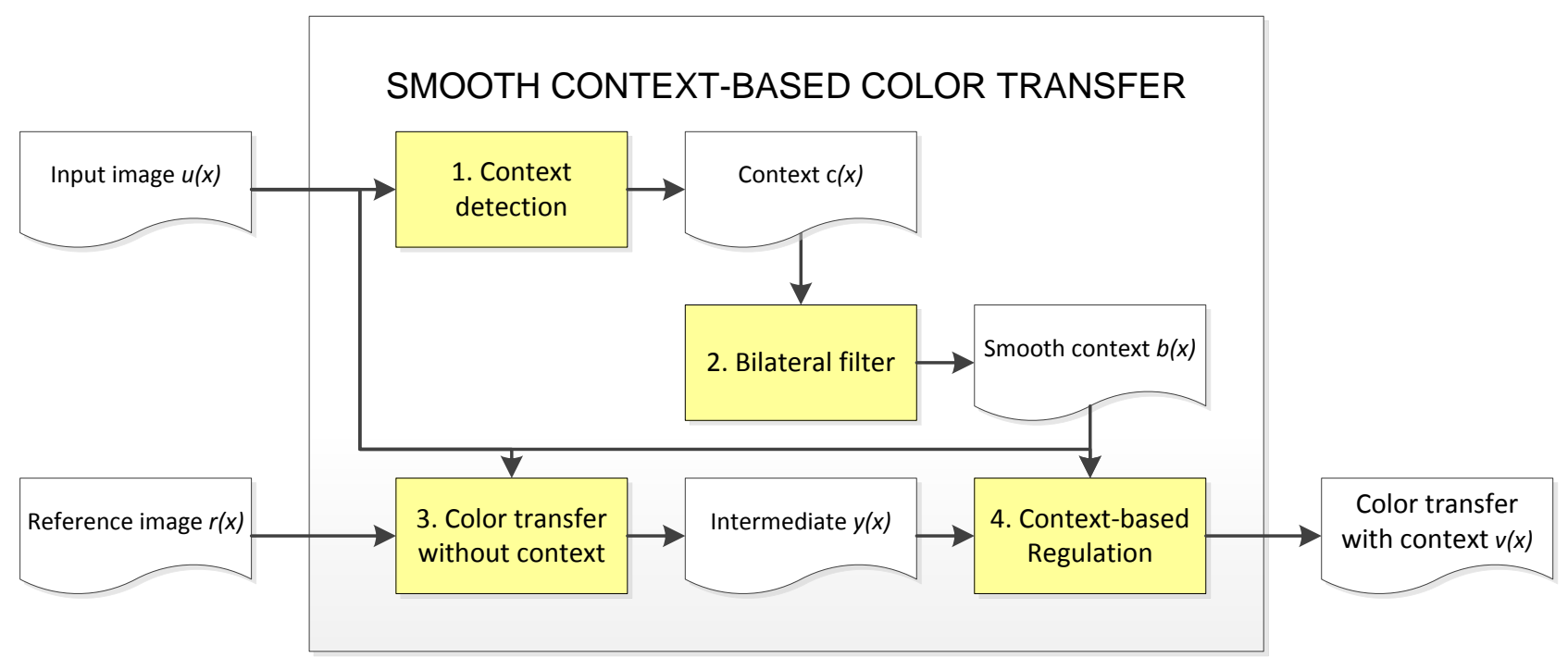

Figure 2:Algorithm of smooth context-based color transfer

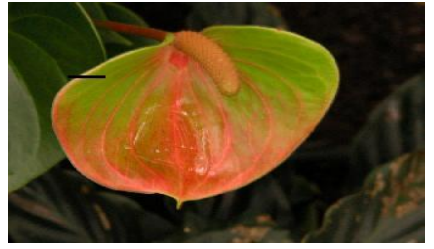

a) Originalmerry0006.jpg

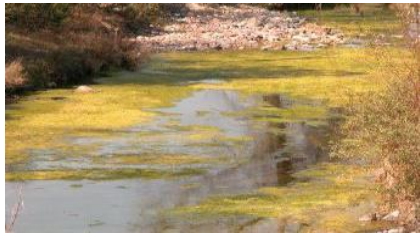

b)Reference color

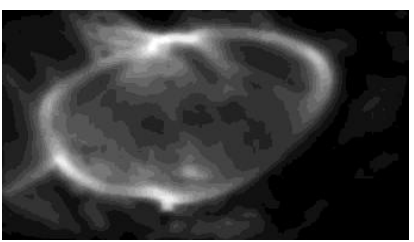

c)Saliency based context

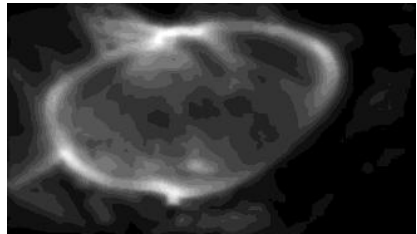

d)Smooth context

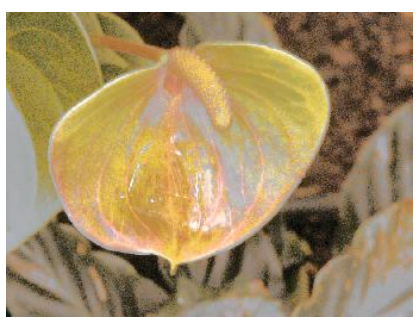

e) Transfer without context, SSIM $=0.9868, t=3.28$

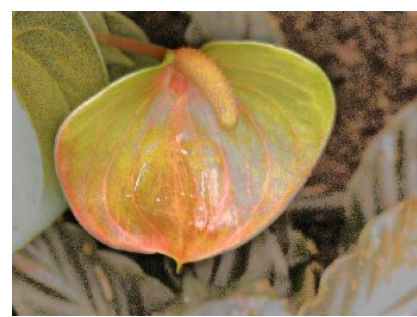

g) Transfer for bacground with context, SSIM=0.9911, $\mathrm{t}=3.28$

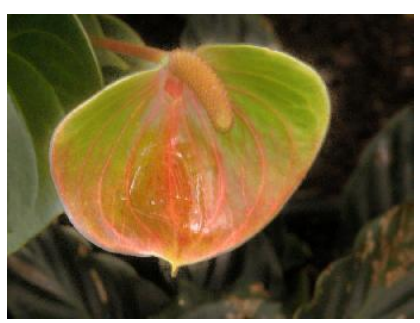

h) Transfer for foreground with context, SSIM=0.9991, $t=3.28$

Figure 3: Example of smooth context-based color transfer 


\subsection{Algorithm}

The algorithm SCCT is short for "Smooth Context-based Color Transfer". It contains the following steps.

Start: given an input image $u(x)$ and reference image $r(x)$. Use (3) to present $u(x)$ and $r(x)$ in Lab.

1. Context detection: defines saliency context $c(x)$ by (7) and (8)[6].

2.Bilateral filter: makes context smooth $b(x) \mathrm{by}(9)$ [9].

3.Transfer without context: transfer full color schema to get $y(x)$ by (10) and (11)[2].

4.Context-based regulation: regulates color transfer proportion by content-related weights $c(x)$ to find $v(x)$ by (12) and (13) for foreground and background accordingly.

Use (4) to get $v(x)$ in RGB.

Figure 2 illustrates four steps of the algorithm by 4 blocks.

\section{EXPERIMENTS}

\subsection{EXAMPLES OF SCCT}

An example is displayed in figure 3: Input color image $u(x)$ (fig.3a) from [10]needs to have new color transferred from reference image $r(x)$ (fig. $3 \mathrm{~b}$ ). Step 1 of the SCCT produced saliency based context $c(x)$ in fig.3c. After step 2 we have smooth context $b(x)$ in fig.3d. Using step 3, full color transferred version $y(x)$ is shown in fig.3e. Finally, step 4 produces two versions $v(x)$ for foreground (fig.3g) and background (fig.3h).Fig. $3 \mathrm{f}$ draws original signal $u(x)$, saliency $s(x)$, target color $r(x)$ and saliency guided context $c(x)$ according to a black line in top left of fig.3a. The saliency guided context is smooth by the bilateral filter.

Each output image is shown with its SSIM index and run time in sec. Some other examples from [10] for foreground transfer are shown in Fig.5. Competitive high level of SSIM index for the examples strongly demonstrated benefits of context in handling color transfer: Result images keep well structure similar to their inputs. Statistics on SSIM average for examples from [10] show that both results with context on foreground and background have SSIM better than color transfer without context, see Fig.4. Background usually has largest space so applying context for them produces the best SSIM index.

Reference images from [24] are selected for visual view with our result as in Fig. 6 to show results of other color transfer methods: separable linear transfer, Cholesky based transfer and linear Monge Kantorovitch transfer.

\subsection{Tone Mapping}

For completeness, in this experimental section the SCCT is tested with some tone mapping examples in Fig.8 from [11]. A part of each image is selected to make reference image for the SCCT algorithm. The parts should have harmonic contrast, so the contrast [25] can be transferred to result with harmonic contrast too. Though this solution is manual in the way of choosing reference image, it supports creative design for new look of media. Fig.7 shows tone mapping from [27], [28], [29] and SCCT for the same input. SCCT gives tone mapping result and keep well image structure with SSIM=1.

\section{DISCUSSION}

From the results reported for different image types we conclude that the SCCT method gives promising way for color transfer keeping initial context and saliency. Intuitively, using context information makes solution easier. In our method, preferable saliency detection method is context aware [2]. The saliency map played context role for color transfer. Bilateral filter makes context smooth to avoid color distortion and grain effect. The filter contains intensive filter with deviation $\sigma_{r}$ to keep closely with local intensive change - the context condition in other words. So our solution is consistent and totally context-based.

\section{FUTURE WORK}

As future work, a new application of color transfer integrated with other features can get attention for improvement of the computer vision task.

\section{CONCLUSION}

A novel method for color transfer is presented. The main contribution is to show that the saliency based context significantly improves on existing methods. The saliency detection is included in the method to generate context for handling transfer process. Imposing this saliency in practices could make color transfer more comfortable with specific applications. Several examples, including tone mapping were shown with high level of accuracy and they demonstrated efficacy and usefulness of the method.

\section{ACKNOWLEDGMENTS}

We thank Erik Reinhard et al [11] for providing for his comprehensive paper and data on Photographic Tone Reproduction for Digital Images. Special thanks to François Pitié for his paper [24] and codes, with these implementation of this work started. We wish to thank Fred Kingdom's Laboratory for the McGill Calibrated Color Image Database [10], its images were used in Fig 3 and Fig 6of this article. Author would also like to thank the anonymous referees for their valuable comments.

\begin{tabular}{|l|r|}
\hline Color transfer & SSIM \\
\hline IDT, no context [02] & 0.9952 \\
\hline Context on foreground & 0.9968 \\
\hline Context on background & 0.9996 \\
\hline
\end{tabular}

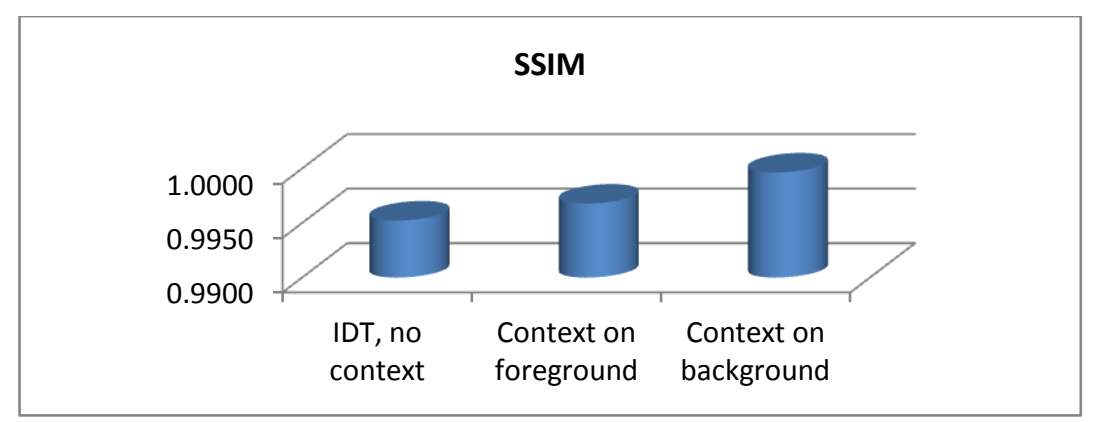


Figure 4: Statistics on SSIM average for examples from [10]
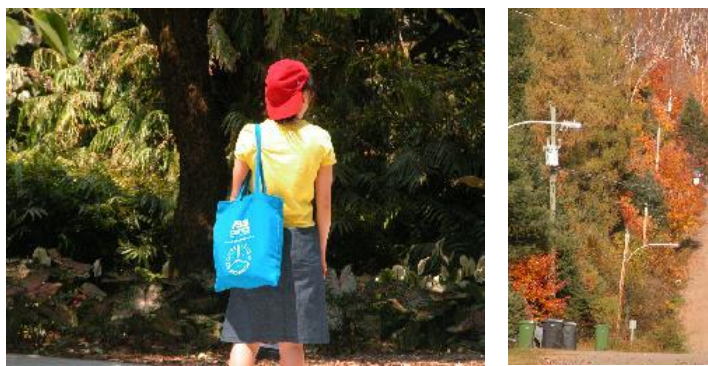

b) Reference

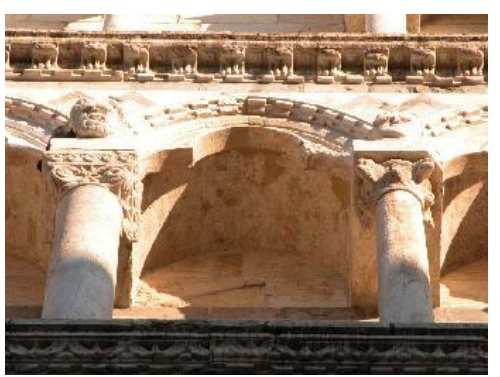

e) merry_italy0044.jpg

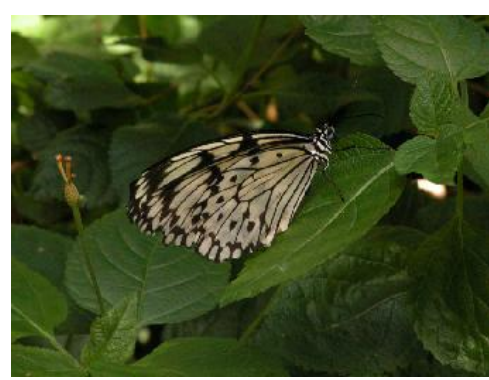

i) pippin0128.jpg

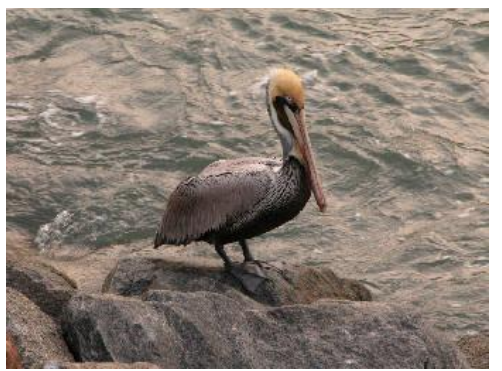

m) merry_mexico0125.jpg

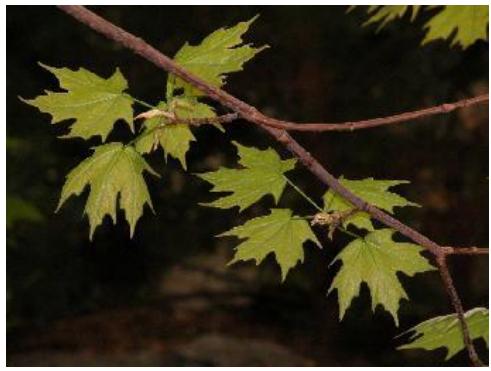

q) pippin0096.jpg

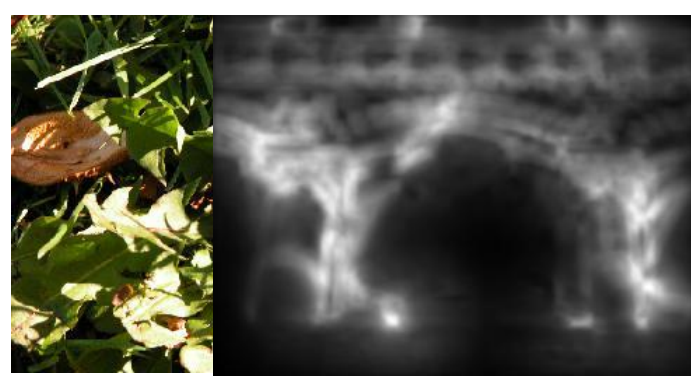

g) Context

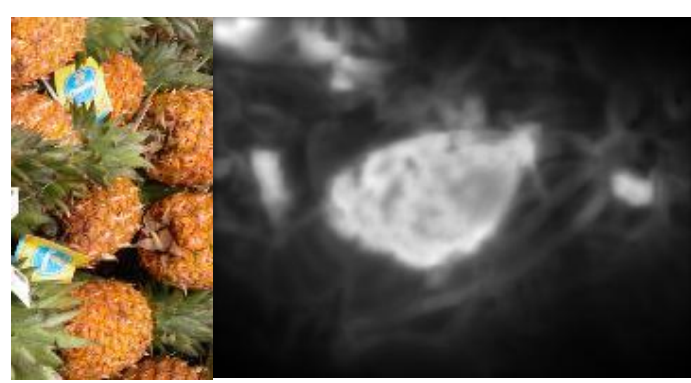

j) Reference

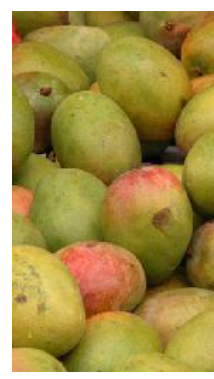

n) Reference

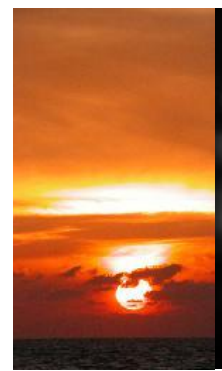

r) Reference

k) Context

o) Context

s) Context

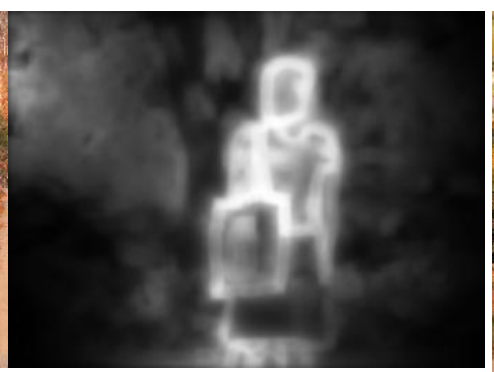

c) Context

d) $\operatorname{SSIM}=0.9962 . t=3.29$

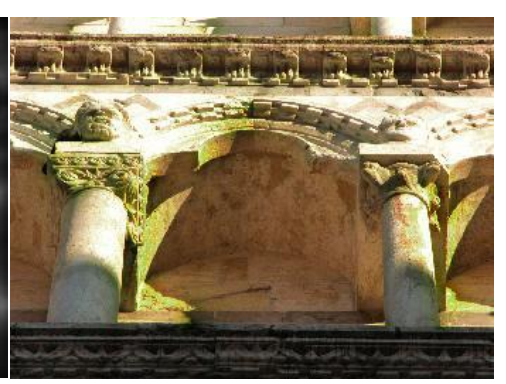

h) $\operatorname{SSIM}=0.9989 . \mathrm{t}=3.24$

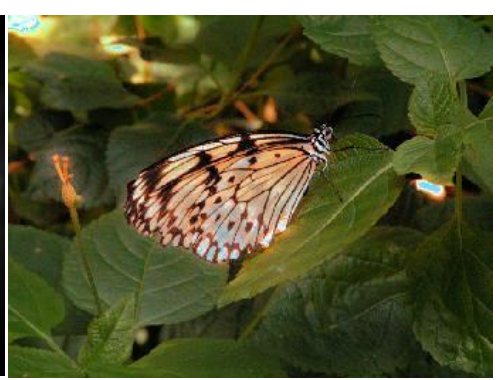

l) $\mathrm{SSIM}=0.9993 . \mathrm{t}=2.92$

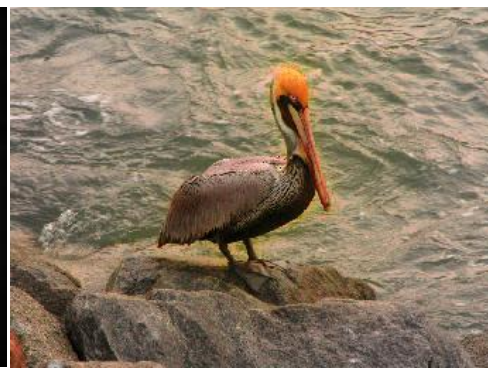

p) $\mathrm{SSIM}=1.0000 . \mathrm{t}=3.32$

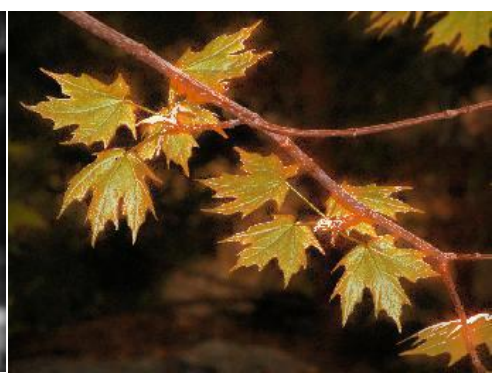

t) $\mathrm{SSIM}=0.9990 . \mathrm{t}=3.16$ 


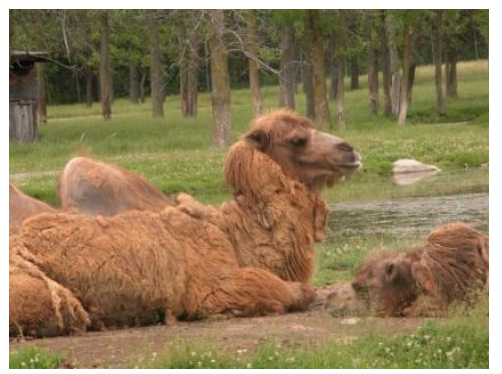

u) pippin0021.jpg

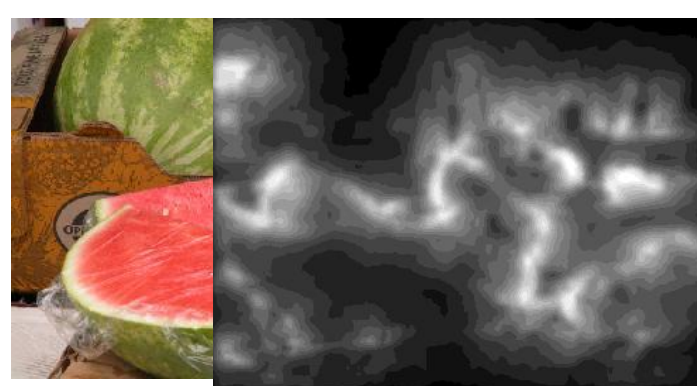

$\begin{array}{ll}\text { v) Reference } & \text { w) Context }\end{array}$

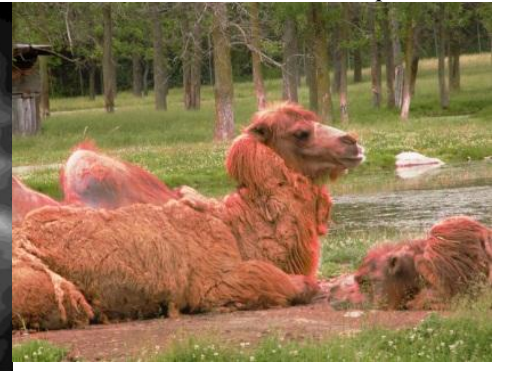

x) $\mathrm{SSIM}=0.9999 . \mathrm{t}=3.30$

Figure 5: Examples of smooth context-based color transfer for color images

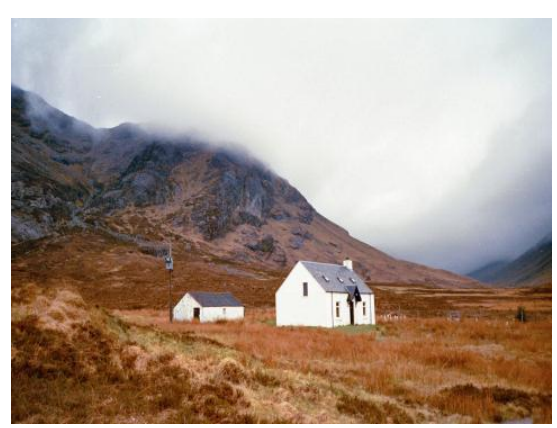

a) Input

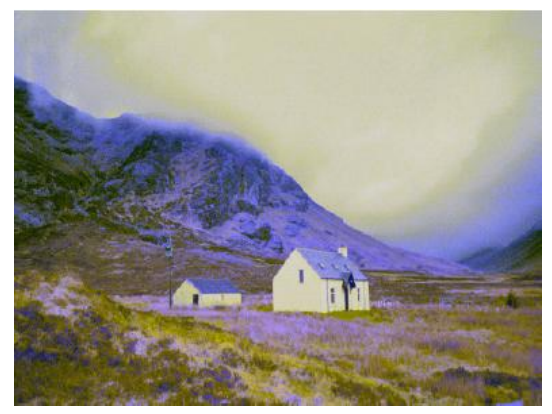

d) Cholesky based transfer

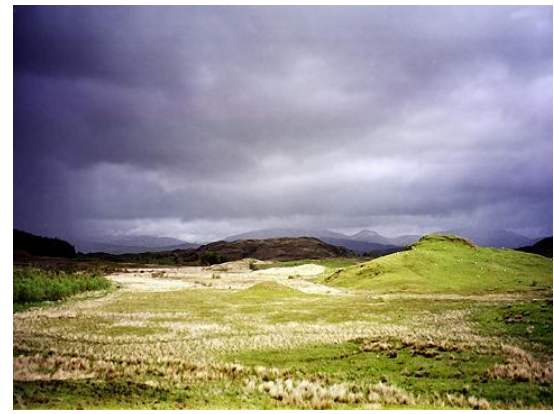

b) Reference

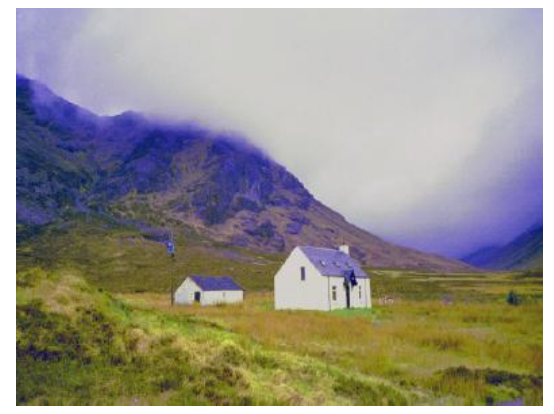

e) Linear Monge-Kantorovitch transfer[24]

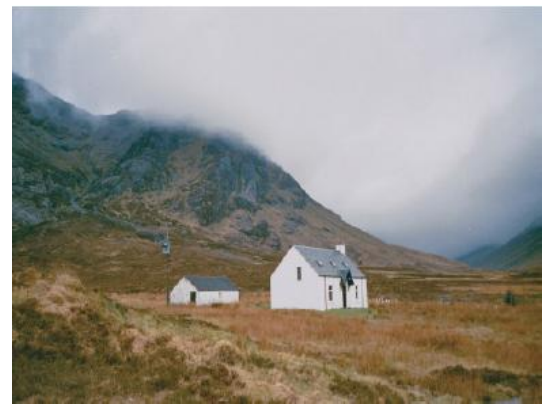

c) Separable linear transfer

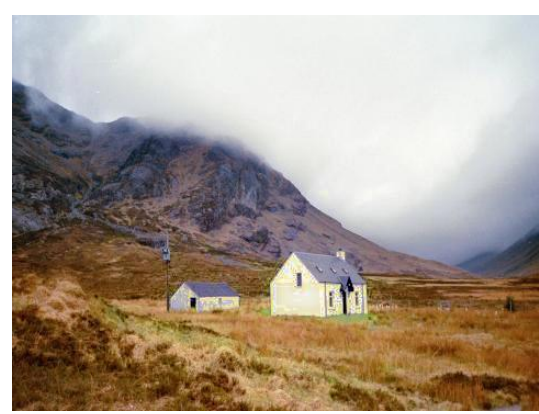

f) SCCT, foreground

Figure 6: Examples of other color transfermethods

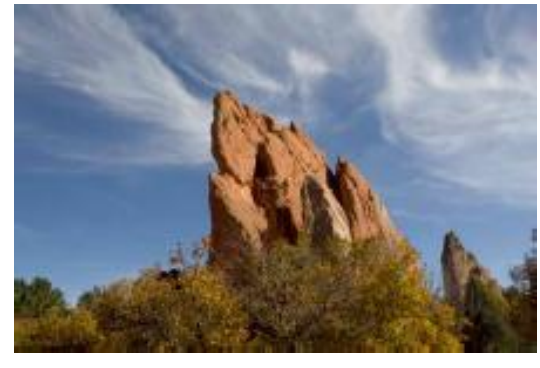

a) input

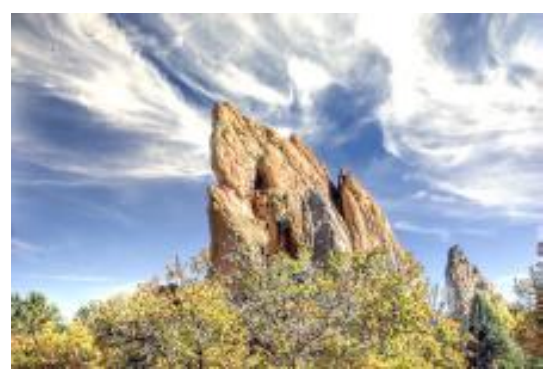

d) Yu-Jui Lin et al [29], SSIM=0.9927

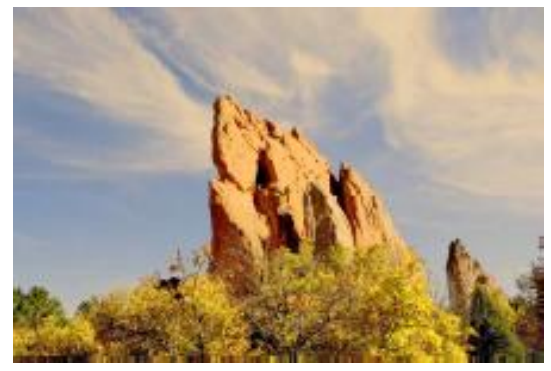

b) Meylan et al [1], SSIM=0.9971

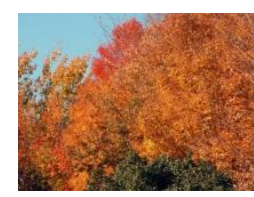

e) Reference image

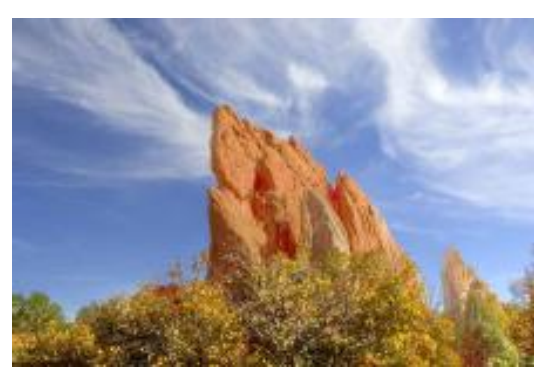

c)Shan et al [28], SSIM=0.9979

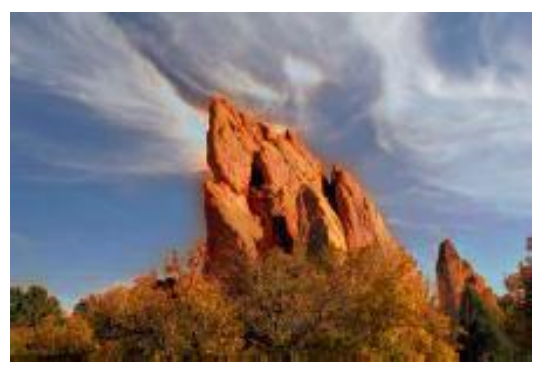

f) $\mathrm{SCCT}$ with reference e), $\mathrm{SSIM}=1$.

Figure 7: Examples of tone mapping from [28], [29] and [30] and SCCT 


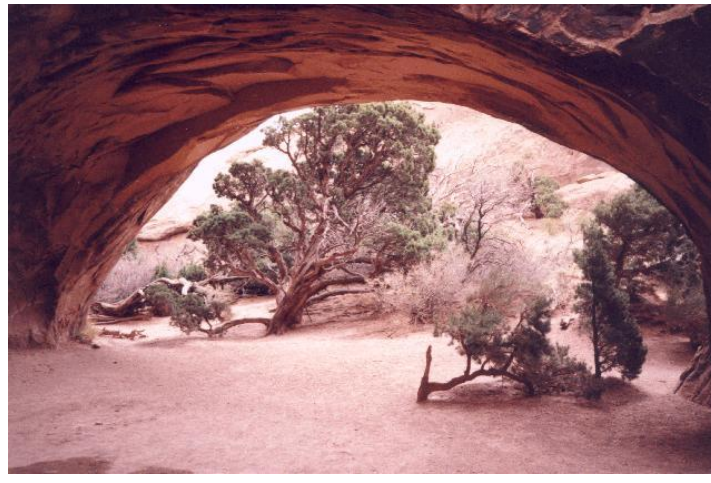

a) Arch.jpg

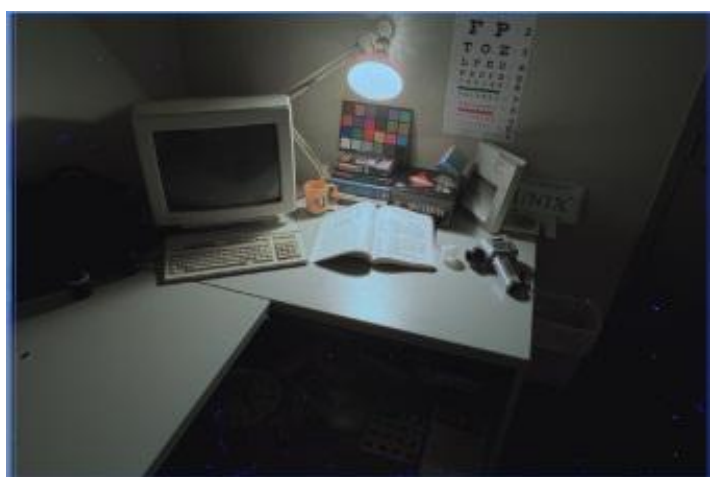

d) Desk.jpg

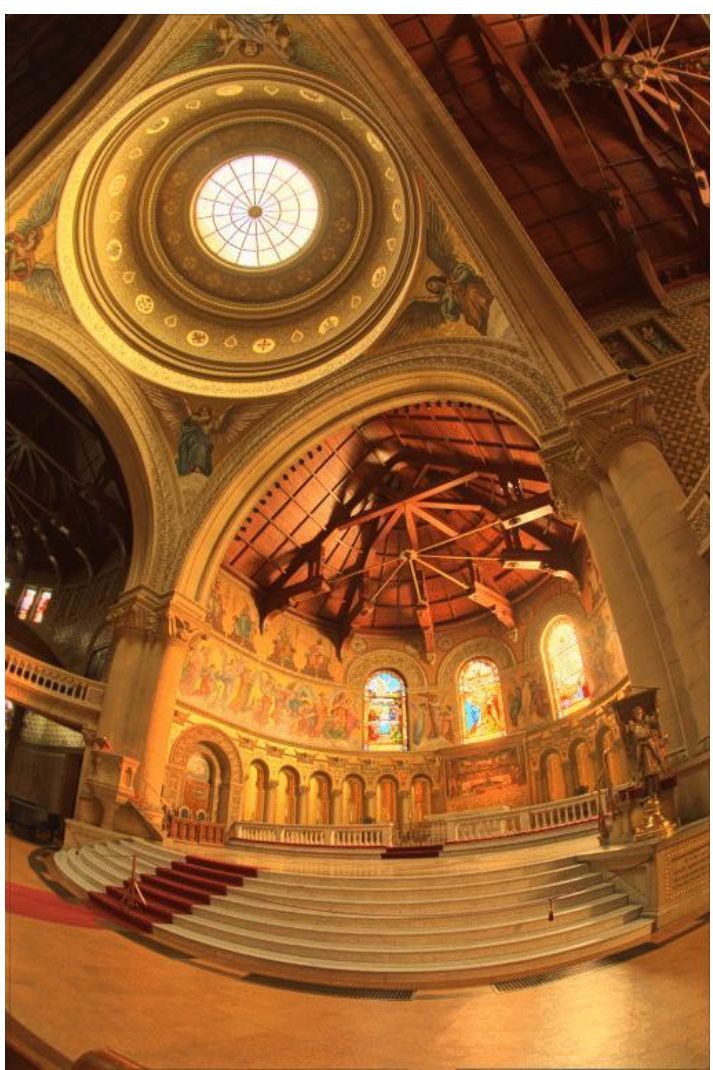

f) Memorial.jpg

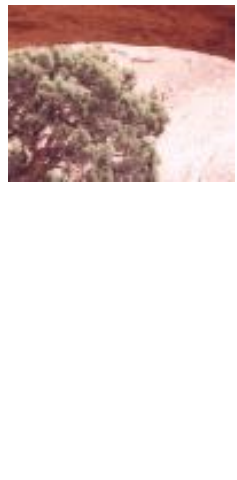

b) Reference
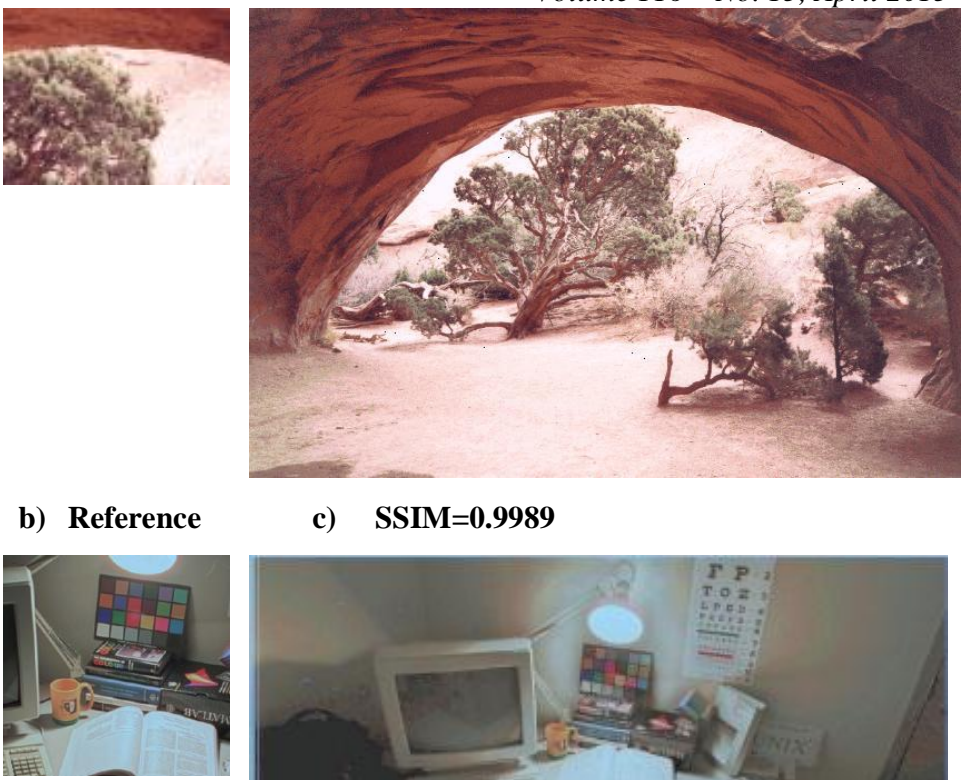

c) SSIM $=0.9989$

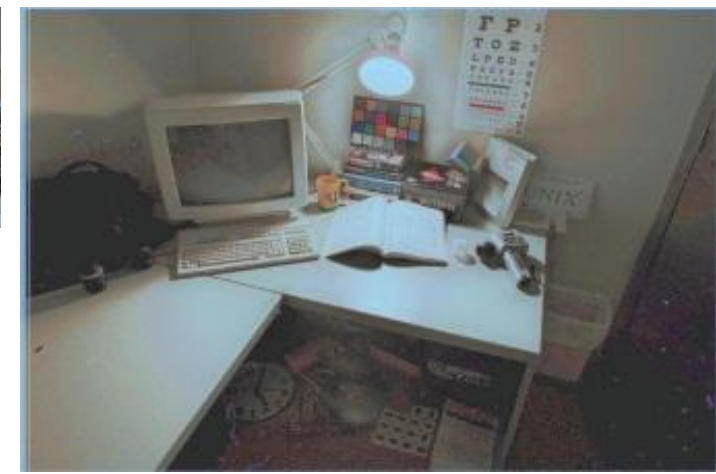

f) $\mathrm{SSIM}=0.9939$
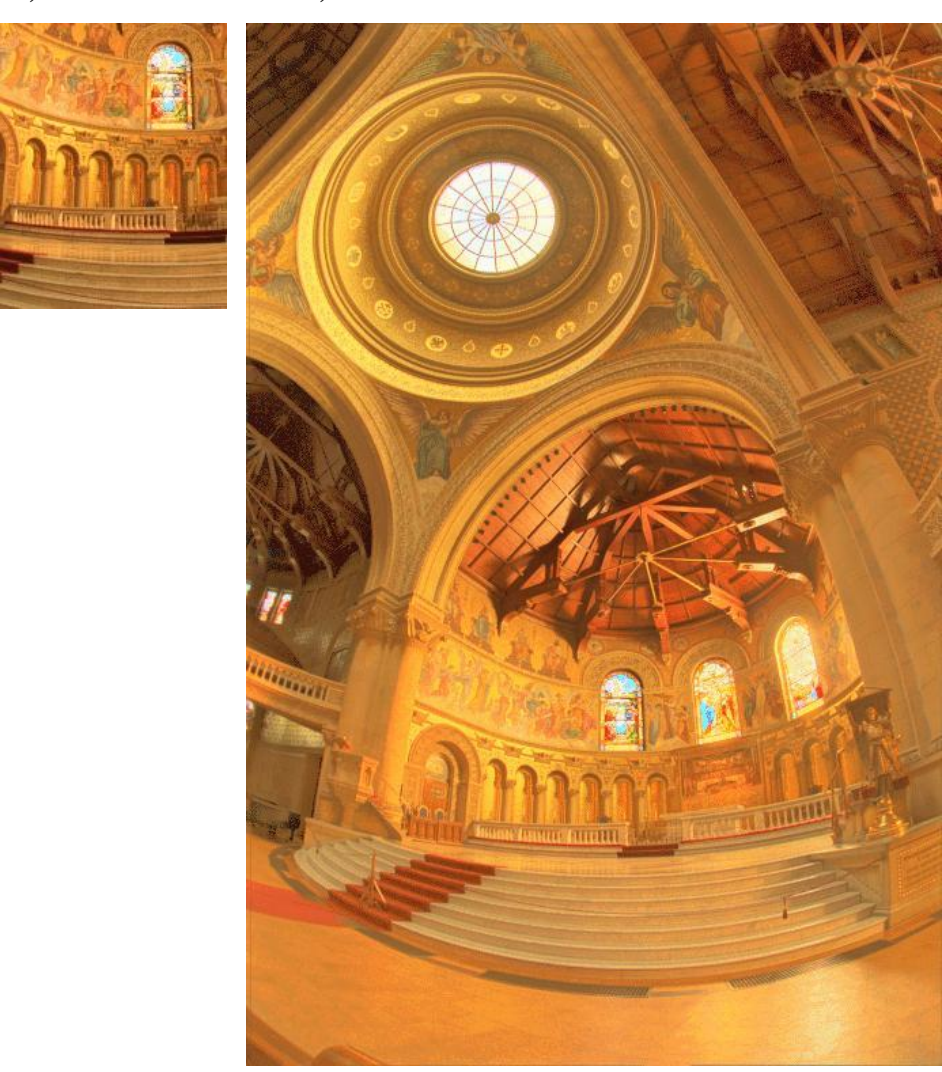

h) $\quad$ SSIM $=0.9976$

g) Reference 


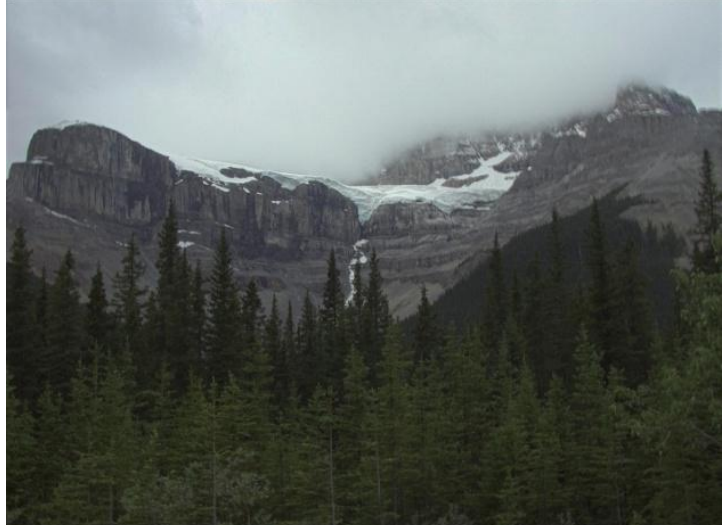

l) crowfoot.jpg

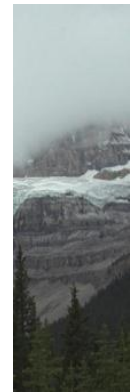

m)Reference

Figure 8: Examples of smooth context-based color transfer for tone mapping

\section{REFERENCES}

[1] E. Reinhard, M. Ashikhmin, B. Gooch, and P. Shirley, Color transfer between images, IIEEE Comput. Graph. Applicat., vol. 21, no. 5, pp.34-41, 2001.

[2] F. Pitié, A. C. Kokaram, and R. Dahyot, N-dimensional probability density function transfer and its application to colour transfer, in Proc. 10th IEEE Int. Conf. Computer Vision, 2005, vol. 2, pp. 1434-1439.

[3] W. Dong, G. Bao, X. Zhang, and J.-C. Paul, -Fast local color transfer via dominant colors mapping, ACM SIGGRAPH Asia 2010Sketches, pp. 46:1-46:2, 2010.

[4] Tania Pouli, Erik Reinhard, Progressive Color Transfer for Images of Arbitrary Dynamic Range, Computers and Graphics 35(1), pp. 67-80, Elsevier, 2011.

[5] Yiming Liu, Michael Cohen, Matt Uyttendaele, Szymon Rusinkiewicz, AutoStyle: Automatic Style Transfer from Image Collections to Users' Images, Eurographics Symposium on Rendering 2014 Wojciech Jarosz and Pieter Peers (Guest Editors) Volume 33 (2014), Num 4.

[6] Stas Goferman, Lihi Zelnik-manor, Ayellet Tal,Contextaware saliency detection (2010), IEEE Conf. on Computer Vision and Pattern Recognition.

[7] Z. Wang, A. C. Bovik, H. R. Sheikh and E. P. Simoncelli, Image quality assessment: From error visibility to structural similarity, IEEE Transactions on Image Processing, vol. 13, no. 4, pp. 600-612, Apr. 2004.

[8] Francois Pitie, Anil C. Kokaram, Rozenn Dahyot, Automated color grading using colour distribution transfer, Computer Vision and Image Understanding, 2007(107), pp.123-137.

[9] S. Paris, P. Kornprobst, J. Tumblin and F. Durand: Bilateral Filtering: Theory and Applications, Computer Graphics and Vision Vol 4, No.1 (2008) 1- 73.

[10] Olmos, A., Kingdom, F. A. A. (2004), A biologically inspired algorithm for the recovery of shading and reflectance images, Perception, 33, 1463 - 1473.

[11] Erik Reinhard, Mike Stark, Peter Shirley and Jim Ferwerda, Photographic Tone Reproduction for Digital Images, SIGGRAPH '02 Proceedings of the 29th annual conference on Computer graphics and interactive techniques, pp. 267-276 ACM, 2002.
[12] R. C. Gonzalez and R. E. Woods, Digital Image Processing, 3rd ed. Upper Saddle River, NJ, USA: Prentice Hall, 2008.

[13] S. Paris, P. Kornprobst, J. Tumblin, and F. Durand,-Bilateral filtering: Theory and application, in Proc. Computer Graphics and Vision 2008.

[14] Zhenhua Li, Zhongliang Jing, Xuhong Yang, Shaoyuan Sun, Color transfer based remote sensing image fusion using non-separable wavelet frame transform, Pattern Recognition Letters 26 (2005) 2006-2014.

[15] Youngbae Hwang, Joon-Young Lee, In So Kweon, Seon Joo Kim, Color, Transfer using Probabilistic Moving Least Squares, IEEE Int Conf on Computer Vision and Pattern Recognition (CVPR), 2014.

[16] K.-Y. Chang, T.-L. Liu, S.-H. Lai. From co-saliency to co-segmentation: An efficient and fully unsupervised energy minimization model. CVPR, pp 2129-2136, 2011.

[17] G. Sharma, F. Jurie, and C. Schmid. Discriminative spatial saliency for image classification. In CVPR, pages 3506-3513, 2012.

[18] P. Hiremath and J. Pujari. Content based image retrieval using color boosted salient points and shape features of an image. International Journal of Image Processing, 2(1):10-17, 2008.

[19] Kohji Kamejima, Saliency-based boundary object detection in naturally complex scenes. RO-MAN 2011: 407-412.

[20] Jiazhi Xia, Saliency-Guided Color Transfer between Images, Advances in Visual Computing, Lec Notes in Computer Science Vol 8033, 2013, pp 468-475.

[21] Arvind Nayak, Subhasis Chaudhuri, and Shilpa Inamdar, Color Transfer and its Applications, Speech, Audio, Image and Biomedical Signal Processing using Neural Networks, Studies in Computational Intelligence Volume 83, 2008, pp 217-241.

[22] Gabriela Csurka, Sandra Skaff, Luca Marchesotti, Craig Saunders, Learning moods and emotions from color combinations. ICVGIP 2010: 298-305.

[23] Kullback, S.; Leibler, R.A. (1'951). On information and sufficiency. Annals of Mathematical Statistics 22 (1): 79-86. doi:10.1214/aoms/1177729694. MR 39968. 
[24] Pitié, F., Kokaram, A.: The Linear Monge-Kantorovitch Color Mapping for Example-Based Color Transfer. In: Proc. of CVMP 2006.

[25] Rabin, J.; CEREMADE, Univ. Paris Dauphine, Paris, France; Delon, J.; Gousseau, Y., Regularization of transportation maps for color and contrast transfer, Image Processing (ICIP), 2010.

[26] D. L. Ruderman, T.W. Cronin, and C. C. Chiao. Statistics of Cone Responses to Natural Images: Implications for Visual Coding. Journal of the Optical Society of America, (8):2036-2045, 1998.
International Journal of Computer Applications (0975 - 8887)

Volume 116-No. 15, April 2015

[27] Meylan, M., Susstrunk, S. 2006. High dynamic range image rendering with a retinex-based adaptive filter. IEEE Trans on Image Proc 15, 9, 2820-2830.

[28] Shan, Q., Jiaya, J.,Brown, M. 2010. Globally optimized linear windowed tone-mapping. IEEE Tran on Visual and Computer Graphics 16, 4 (July), 663-675.

[29] Yu-Jui Lin, Chih-Tsung Shen, Chun-Cheng Lin, HsuChun Yen, Edge-Preserving Image Decomposition using L1 Fidelity with L0 Gradient, SIGGRAPH Asia 2012 Tech Briefs (SA '12). 